\title{
Fiber-Optic Cavity Ring Down Using an Added-Signal for Curvature Sensing
}

\author{
S. Silva, P. Biswas, S. Bandyopadhyay, P. A. Jorge, M. B. Marques, and O. Frazão
}

\begin{abstract}
This letter presents a fiber-optic cavity ring down (CRD) configuration using an added-signal for curvature sensing. An optical time-domain reflectometer was used to send impulses down into the fiber loop cavity, inside of which a long period grating was placed to act as a sensing device. The added-signal was obtained by the sum of several conventional CRD impulses, thus providing an improvement on the curvature sensitivity when compared with the conventional CRD signal processing. A linear response to applied curvature in the range of 2.2-3.6 $\mathrm{m}^{-1}$ was observed, and a sensitivity of $15.3 \mu \mathrm{s} / \mathrm{m}^{-1}$ was obtained. This result was found to be 20 -fold the one obtained for the conventional CRD signal processing. The added-signal increases the optical power but increases as well the ring-down time due to the sum of the several loops that light travels inside the ring. A ring-down time response of $43.3 \mu \mathrm{s}$ was attained (versus 23.7 $\mu$ s for the conventional CRD signal processing).
\end{abstract}

Index Terms-Cavity ring down, curvature, long period grating, OTDR, optical fiber sensors.

\section{INTRODUCTION}

$\mathbf{L}$ ONG period gratings (LPGs) are well-known fiber devices that have been used for a wide range of sensing applications [1]. In 1998, Lee and Nishii [2] reported the curvature sensitivity of a pair of in-series LPGs. It was shown that the interference fringes formed by the LPG pair were sensitive to the loss in the cladding mode, which could be induced by bending the fiber. Later, LPGs written in commercially available boron-codoped fibers were shown to exhibit high temperature and bending sensitivities [3]. For an LPG bent in the curvature range of $0.4-0.8 \mathrm{~m}^{-1}$, sensitivities

Manuscript received January 20, 2015; revised May 5, 2015; accepted May 10, 2015. Date of publication July 10, 2015; date of current version August 31, 2015. This work was supported in part by the Fundação para a Ciência e Tecnologia (Portuguese Foundation for Science and Technology) in part by the European Regional Development Fund (ERDF) through the COMPETE Programme (Operational Programme for Competitiveness) within Project FCOMP-01-0124-FEDER-037281, and in part by the Indo-Portuguese Research Cooperation in Science and Technology. The work of S. Silva was supported in part by the European Union and in part by the Portuguese State under Grant SFRH/BPD/92418/2013.

S. Silva and P. A. Jorge are with the Instituto de Engenharia de Sistemas e Computadores, Tecnologia e Ciência, Porto 4169-007, Portugal (e-mail: sfsilva@inescporto.pt; pjorge@inescporto.pt).

P. Biswas and S. Bandyopadhyay are with the Fiber Optics and Photonics Division, Council of Scientific and Industrial Research-Central Glass and Ceramic Research Institute, Kolkata 700032, India (e-mail: palas@cgcri.res.in; somnath@cgcri.res.in).

M. B. Marques and O. Frazão are with the Instituto de Engenharia de Sistemas e Computadores, Tecnologia e Ciência, Porto 4169-007, Portugal, and also with the Department of Physics and Astronomy, Faculty of Sciences, University of Porto, Porto 4169-007, Portugal (e-mail: mbmarque@fc.up.pt; ofrazao@inescporto.pt).

Color versions of one or more of the figures in this letter are available online at http://ieeexplore.ieee.org.

Digital Object Identifier 10.1109/LPT.2015.2453124 of $11.2 \mathrm{~nm} / \mathrm{m}^{-1}$ and $-14.0 \mathrm{~nm} / \mathrm{m}^{-1}$ were attained for the two attenuation bands. A few years later, Frazão et al. [4] reported an alternative technique to interrogate an LPG: to read the amplitude changes with applied curvature, a conventional optical time domain reflectometer (OTDR) was used. In the curvature range between 2.0 and $5.5 \mathrm{~m}^{-1}$, a sensitivity of $2.16 \mathrm{~dB} / \mathrm{m}^{-1}$ was obtained. Meanwhile, the same research group produced a Mach-Zehnder curvature sensor based on a Singlemode-multimode-singlemode (SMS) fiber layout, where the multimode interference phenomenon was combined with the operation of an LPG [5]; in the curvature range of $0.1-1.6 \mathrm{~m}^{-1}$, the SMS fiber sensor exhibited a maximum sensitivity of 612.4 degrees $/ \mathrm{m}^{-1}$. Wang and Rao [6] demonstrated that the UV laser-induced LPG is independent of the bend-directions, while the bend-sensitivity of $\mathrm{CO}_{2}$ laserinduced LPFGs is strongly dependent on the curved directions. Jin et al. [7] presented instead a directional bend sensor based on an LPG formed by introducing periodic grooves along one side of a photonic crystal fiber (PCF) with a focused $\mathrm{CO}_{2}$ laser beam. A bend sensitivity of $2.26 \mathrm{~nm} / \mathrm{m}^{-1}$ within the range of -5 to $5 \mathrm{~m}^{-1}$ was demonstrated. Recently, LPGs have been fabricated in a side-hole fiber (SHF) by using a pulsed $\mathrm{CO}_{2}$ laser [8]. Results have shown to possess a bend-direction-dependent sensitivity up to $9.36 \mathrm{~nm} / \mathrm{m}^{-1}$, within the range 0.644 to $1.288 \mathrm{~m}^{-1}$. In a different perspective, Wang et al. [9], proposed a fiber device based on overlapping an LPG on a fiber Bragg grating (FBG), which have shown to be capable of measuring directional bending and temperature. Experimental results have shown that the bending sensitivity was $-6.819 \mathrm{~nm} / \mathrm{m}^{-1}$ in the range of $2-2 \mathrm{~m}^{-1}$.

Cavity ring-down (CRD) spectroscopy is a well-established technique that gained popularity with the demonstration of highly sensitive direct absorption measurements with pulsed light sources [10]. The combination of optical fibers and the CRD scheme has shown significant potential in fiber-optic sensing applications, namely, for the measurement of strain [11], pressure [12], temperature [13], refractive index [14] and biochemical sensing [15]. von Lerber and Sigrist [16] reported a CRD scheme that enabled measurements of optical losses in high-finesse fiber-optic cavities induced by fiber bending.

This work presents a fiber-optic ring down system that uses an OTDR as modulated optical source and an LPG inside the fiber loop as sensing device. The LPG was interrogated using (1) the CRD conventional signal processing and (2) when an added-signal was applied in the fiber loop. In the second case, the added-signal was obtained by the sum of 


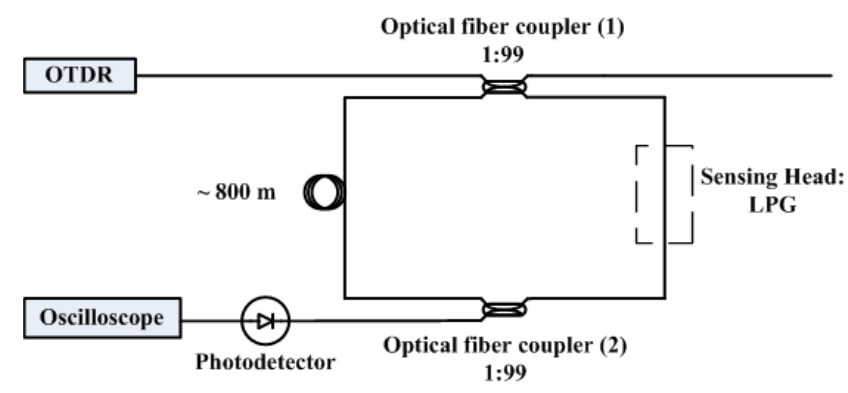

Fig. 1. Experimental setup of the proposed CRD that uses an LPG as sensing head. The optical modulated source is an OTDR that launches the input signal inside the fiber cavity; the output signal is monitored by means of a photodetector and an oscilloscope.



Fig. 2. Optical spectrum of the LPG used as sensing head in the proposed CRD configuration.

several conventional CRD impulses. The LPG was submitted to curvature in these two distinct cases.

\section{Curvature Sensing}

The experimental setup of the proposed CRD system is presented in Figure 1. The cavity is composed of two standard 1:99 optical fiber couplers $(2 \times 1)$, a fiber loop with $\sim 800 \mathrm{~m}$ (SMF 28) and an LPG as sensing device. A commercial OTDR (Yokogawa AQ7270) is used to send impulses down into the fiber cavity, instead of the usual laser and modulator setup [17]. The train of pulses is coupled via $1 \%$ arm of the input optical coupler, rings around inside the fiber loop and is coupled out via $1 \%$ arm of the output coupler; the amplitude of the output pulses decay temporally due to the total existing losses in the fiber loop (fiber loss, fiber couplers insertion losses, LPG transmission attenuation), passes through a photodetector (gain of $50 \mathrm{~dB}$ ) and is monitored in an oscilloscope. The output signal of the OTDR is used only as optical modulated source [18]. The advantage of using this equipment is to allow system optimization either in terms of impulse and optical power - allowing its use in long distance applications. Furthermore, the use of a multimode laser source increases the optical power inside the fiber loop [19].

The optical spectrum of the LPG placed inside the fiber loop cavity is presented in Figure 2. The LPG was written by UV in SMF28e with a period of $335 \mathrm{~nm}$ and a length

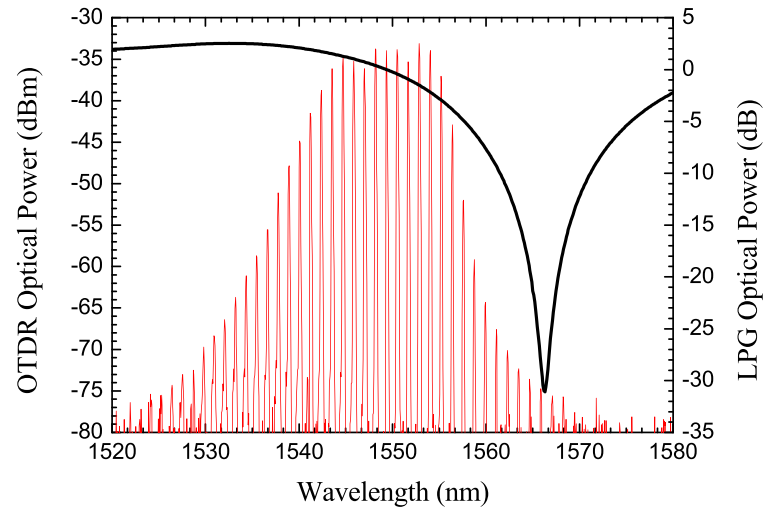

Fig. 3. Overlap of the LPG optical spectrum with the OTDR multimode laser source.

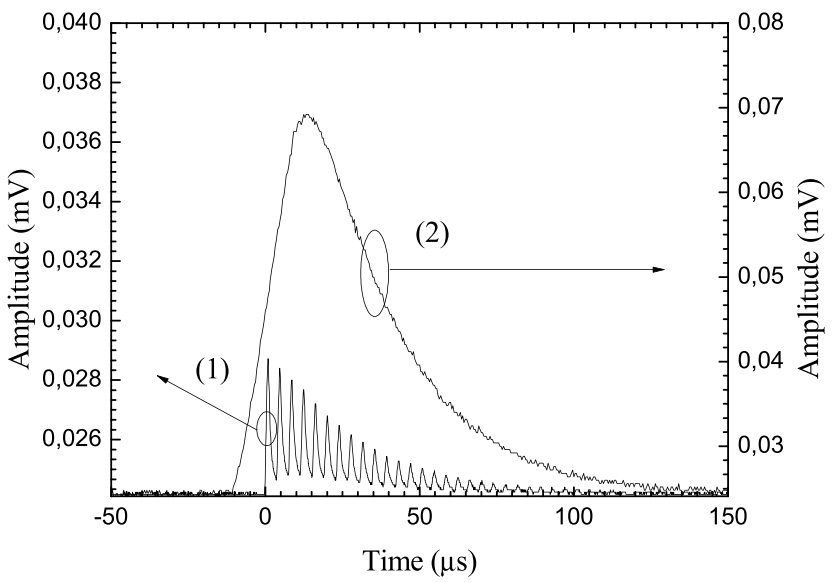

Fig. 4. Cavity ring down trace for (1) conventional configuration and (2) added-signal obtained by the sum of several conventional CRD impulses.

of $25 \mathrm{~mm}$. The $\mathrm{LP}_{07}$ resonance mode of the LPG is positioned at approximately $1566.2 \mathrm{~nm}$ with a full width half maximum (FWHM) of $33.7 \mathrm{~nm}$. This resonance is situated in the long-wavelength side of the multimode laser spectrum which is centered at $1550 \mathrm{~nm}$ and has a bandwidth of $50 \mathrm{~nm}$, as shown in Figure 3.

Figure 4 shows the cavity ring-down trace for the two cases when the LPG was interrogated using (1) the CRD conventional signal processing and (2) when an added-signal was applied in the fiber loop. In the first case (1), the OTDR sent impulses of $1 \mu \mathrm{s}$ at $1550 \mathrm{~nm}$ down into the fiber cavity; the time of a single round trip is ca. $3.85 \mu \mathrm{s}$ and is determined by the cavity length. An exponential fit was also performed and a ring-down time of $23.7 \mu$ s was attained, with an associated loop loss of $0.7 \mathrm{~dB}$. In the second case (2), the OTDR sent impulses of $20 \mu \mathrm{s}$ at $1550 \mathrm{~nm}$ in order to obtain the addedsignal. After performing an exponential fit, a ring-down time of $43.3 \mu$ s was obtained. Such results show that the addedsignal increases the optical power but increases as well the ring-down time due to the sum of the several loops that light travels inside the ring.

A numerical analysis was performed to better understand the obtained added-signal. Figure 5 presents a simplified model concerning the sum of the several signals acquired by a typical OTDR impulse. This impulse is normally 


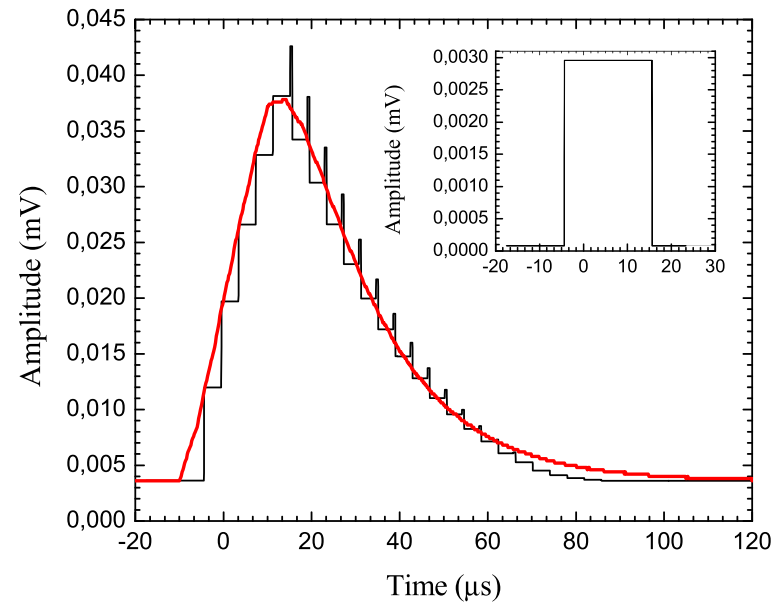

Fig. 5. Numerical analysis and experimental result of the added-signal. Inset shows a single square impulse $(20 \mu \mathrm{s})$ generated by the OTDR.

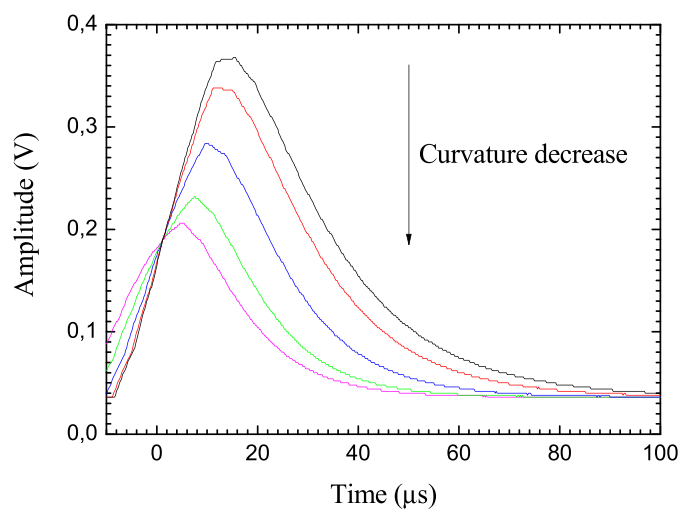

Fig. 6. Cavity ring-down trace obtained when an added-signal is applied in the fiber loop and its behavior when curvature is applied to the LPG.

a well-defined square pulse (in this case, $20 \mu \mathrm{s}$ ) - please see inset of Figure 5. The added-signal starts by the sum of the first impulse with the second one, having a delay time of $3.85 \mu \mathrm{s}$ and an attenuation obtained by the loss inside the fiber loop $(0.7 \mathrm{~dB})$ in the second round. Therefore, the sum of all impulses, i.e. the added-signal, results in a step-like signal as obtained in the numerical analysis depicted in Figure 5. The step shape of this signal disappears when the addedsignal is observed experimentally by the photodetector due to the intrinsic behavior of this component. The comparison between numerical analysis and experimental results shows a good agreement of the obtained added-signals.

The behavior of this structure as a curvature sensor was duly characterized. The fiber was fixed at two points, at a distance of $250 \mathrm{~mm}$ from each other; one of these points is a translation stage that allows the fiber to bend. The bending displacement, $d$, was applied to the LPG via sequential $100 \mu \mathrm{m}$ displacements. Hence, the curvature $(1 / R)$ of the fiber structure changed by $2 d /\left(d^{2}+L^{2}\right)$, where $L$ is half the distance between the two fixed points. Curvature was then applied to the LPG in the two distinct situations presented previously. The results for the added-signal configuration are depicted in Figure 6. The amplitude of the added-signal



Fig. 7. Ring-down time versus curvature applied to the LPG when interrogated by the conventional CRD signal processing and added-signal.

decreases with decreasing curvature, as result of bending the LPG. For similar reasons to those reported in [4], it is expected that the proposed CRD system will also have a low crosssensitivity to temperature. The ring-down time as a function of curvature was also determined for both conventional CRD- and added-signals, as depicted in Figure 7. Results show that the sensitivities obtained for the added-signal and conventional CRD signal are $15.3 \mu \mathrm{s} / \mathrm{m}^{-1}$ and $0.74 \mu \mathrm{s} / \mathrm{m}^{-1}$, respectively. The added-signal configuration presents a sensitivity 20-fold the one obtained for the conventional CRD signal processing.

\section{CONCLUSION}

An optical fiber CRD configuration was proposed, making use of an added-signal for curvature sensing. An OTDR was used to send impulses down into the fiber loop cavity, inside of which an LPG was placed to act as sensing device. The addedsignal was obtained by the sum of several conventional CRD impulses, which provided an improvement on the curvature sensitivity when compared to the conventional CRD signal processing. Curvature was applied to the LPG in the range of 2.2 to $3.6 \mathrm{~m}^{-1}$ and a sensitivity of $15.3 \mu \mathrm{s} / \mathrm{m}^{-1}$ was obtained for the added-signal processing. This values was found to be 20-fold the one obtained for the conventional CRD signal processing $\left(0.74 \mu \mathrm{s} / \mathrm{m}^{-1}\right)$. Since the LPG is interrogated in terms of intensity variations, it is possible to eliminate the common problem of cross-sensitivity to temperature. Also, the CRD system is a low-cost configuration when compared to the usual interrogation technique using an optical spectrum analyzer.

\section{REFERENCES}

[1] S. W. James and R. P. Tatam, "Optical fibre long-period grating sensors: Characteristics and application," Meas. Sci. Technol., vol. 14, pp. R49-R61, Mar. 2003.

[2] B. H. Lee and J. Nishii, "Bending sensitivity of in-series long-period fiber gratings," Opt. Lett., vol. 23, no. 20, pp. 1624-1626, Oct. 1998.

[3] C. C. Ye, S. W. James, and R. P. Tatam, "Simultaneous temperature and bend sensing with long-period fiber gratings," Opt. Lett., vol. 25, no. 14, pp. 1007-1009, 2000.

[4] O. Frazão, R. Falate, J. M. Baptista, J. L. Fabris, and J. L. Santos, "Optical bend sensor based on a long-period fiber grating monitored by an optical time-domain reflectometer," Opt. Eng. Lett., vol. 44, no. 11, pp. 110502-1-110502-3, Nov. 2005. 
[5] O. Frazão et al., "All-fiber Mach-Zehnder curvature sensor based on multimode interference combined with a long-period grating," Opt. Lett. vol. 32, no. 21, pp. 3074-3076, Nov. 2007.

[6] Y.-P. Wang and Y.-J. Rao, "A novel long period fiber grating sensor measuring curvature and determining bend-direction simultaneously," IEEE Sensors J., vol. 5, no. 5, pp. 839-843, Oct. 2005.

[7] L. Jin, W. Jin, and J. Ju, "Directional bend sensing with a $\mathrm{CO}_{2}$-laserinscribed long period grating in a photonic crystal fiber," J. Lightw. Technol., vol. 27, no. 21, pp. 4884-4891, Nov. 1, 2009.

[8] Y. Xin, X. Dong, J. Yuan, Y. Li, S. Jin, and S. Zhang, "Sensing characteristics of side-hole fiber-based long-period grating," Adv. Mater. Sci. Eng., vol. 2013, Jul. 2013, Art. ID 850293.

[9] L. Wang et al., "Simultaneous directional bending and temperature measurement with overlapping long period grating and fiber Bragg grating structure," J. Opt., vol. 16, p. 055401 , Mar. 2014.

[10] A. O'Keefe and D. A. G. Deacon, "Cavity ring-down optical spectrometer for absorption measurements using pulsed laser sources," Rev. Sci. Instrum., vol. 59, pp. 2544-2551, Dec. 1988.

[11] P. B. Tarsa, D. M. Brzozowski, P. Rabinowitz, and K. K. Lehmann, "Cavity ringdown strain gauge," Opt. Lett., vol. 29, no. 12 , pp. 1339-1341, Jun. 2004.
[12] C. Wang and S. T. Scherrer, "Fiber ring-down pressure sensors," Opt. Lett., vol. 29, no. 4, pp. 352-354, Feb. 2004.

[13] C. Wang, "Fiber ringdown temperature sensor," Opt. Eng. Lett., vol. 44, no. 3, p. 030503 , Mar. 2005.

[14] N. Ni, C. C. Chan, L. Xia, and P. Shum, "Fiber cavity ring-down refractive index sensor," IEEE Photon. Technol. Lett., vol. 20, no. 16, pp. 1351-1353, Aug. 15, 2008.

[15] P. B. Tarsa, A. D. Wist, P. Rabinowitz, and K. K. Lehmann, "Single-cell detection by cavity ring-down spectroscopy," Appl. Phys. Lett., vol. 85, no. 19, pp. 4523-4525, Nov. 2004.

[16] T. von Lerber and M. W. Sigrist, "Cavity-ring-down principle for fiber-optic resonators: Experimental realization of bending loss and evanescent-field sensing," Appl. Opt., vol. 41, no. 18, pp. 3567-3575, Jun. 2002.

[17] M. Fabian, E. Lewis, T. Newe, and S. Lochmann, "Optical fibre cavity for ring-down experiments with low coupling losses," Meas. Sci. Technol., vol. 21, p. 094034, Jul. 2010.

[18] D. J. Passos, S. O. Silva, J. R. A. Fernandes, M. B. Marques, and O. Frazão, "Fiber cavity ring-down monitoring with an optical timedomain reflectometer," Photon. Sensors, vol. 4, no. 4, pp. 295-299, 2014.

[19] G. Stewart, K. Atherton, and B. Culshaw, "Cavity-enhanced spectroscopy in fiber cavities," Opt. Lett., vol. 29, no. 5, pp. 442-444, Mar. 2004. 